\title{
Testing space weathering models on A-type asteroid (1951) Lick (Research Note)
}

\author{
R. Brunetto ${ }^{1,2}$, J. de León ${ }^{3}$, and J. Licandro ${ }^{3,4}$
}

\author{
1 Dipartimento di Fisica, Università del Salento, via Arnesano, 73100 Lecce, Italy \\ 2 INAF - Osservatorio Astrofisico di Catania, via S. Sofia 78, 95123 Catania, Italy \\ e-mail: rosario.brunetto@le.infn.it \\ ${ }^{3}$ Instituto de Astrofísica de Canarias, c/Vía Láctea s/n, 38205 La Laguna, Tenerife, Spain \\ ${ }^{4}$ Isaac Newton Group, 38700, Santa Cruz de La Palma, Tenerife, Spain
}

Received 26 April 2007 / Accepted 15 June 2007

\section{ABSTRACT}

\begin{abstract}
Aims. A-type asteroid (1951) Lick has a very red visible and near-infrared reflectance spectrum, and it is one of the reddest NearEarth objects. Its surface is probably affected by heavy space weathering processes. Here we investigate the possibility of a weathering saturation regime.

Methods. We compare Lick's spectrum with those of silicate olivine after laser ablation experiments, reported by Brunetto et al. (2006a, Icarus, 180, 546; 2007, Icarus, in press). We apply scattering and space weathering models developed by Hapke (1981, J. Geophys. Res., 86, 3039; 2001, J. Geophys. Res., 106, 10039).

Results. We successfully fit the spectrum of Lick, in terms of slope, band profile, and albedo. Results confirm that Lick has olivine with $\mathrm{Mg}$ number higher than that of the Brachina meteorite. The use of metallic iron inclusions in the model yields a product of volume fraction of inclusions times effective optical path length of about $0.039 \mu \mathrm{m}$, similarly to what observed experimentally after UV laser ablation of Mg-rich olivine.

Conclusions. The surface of Lick is extremely weathered, indicating a saturation regime similar to what observed in the laboratory, and suggesting that the asteroid surface is probably older than about $10^{7}-10^{8}$ years.
\end{abstract}

Key words. minor planets - asteroids - solar system: general

\section{Introduction}

In the Solar System, the surface of asteroids is continuously affected by the interaction with micro-meteorites, solar wind particles, and high energy particles (Chapman 2004). This interaction, known as space weathering, causes several effects. In particular it induces variations in the optical properties of the surface materials. This is revealed by the variations of the spectral properties (Hapke 2001).

In the interpretation of asteroid spectra, an important role is played by laboratory experiments, that simulate the space weathering processes and the corresponding spectral variations. Solar wind and micro-meteorite bombardment have been simulated using ion irradiation and laser ablation respectively (Sasaki et al. 2001; Hapke 2001; Brunetto et al. 2006a,b): increased processing generally results in a redder spectral slope, a lower albedo, and weaker absorption features.

Several models have been developed to characterize the spectra of asteroids in terms of a weathering component and a pristine mineralogical composition, and in some instances to estimate the age of the surface (Hiroi \& Sasaki 2001; Hapke 2001; Brunetto et al. 2006b). In particular, Hapke (2001) developed a model to describe space weathering, considering the formation of metallic iron particles (smaller than the wavelength) in vapordeposited coatings on soil particle surfaces and inside agglutinates. This process has been studied observing the direct link between abundance of surface deposits of nanophase iron and systematic variations of optical properties of lunar soils (Pieters et al. 2000), and by several laboratory results showing the formation of iron nanoparticles in space weathering experiments (Sasaki et al. 2001; Bentley et al. 2005; Brunetto et al. 2006a).

Asteroid (1951) Lick is a Near-Earth object that orbits in the limit that separates Amor objects from Mars Crossers. It has been classified as an A-type asteroid by Bus \& Binzel (2002). Atype asteroids, usually found in the main asteroid belt between Mars and Jupiter, have spectra that are very similar to spectra of olivine (Cruikshank \& Hartmann 1984).

Lick has a very red spectrum (de León et al. 2004), and it is probably one of the most weathered Near-Earth asteroids. Thus, it is interesting to apply the space weathering models mentioned above, as a test for space weathering to the highest levels, and to investigate the saturation regime. A proper estimation of the weathering contribution can be very useful to better constrain the surface composition and age.

\section{Observations and experiments}

The spectral observations of Lick are described by de León et al. (2004). The experiments used in this paper as a simulation of weathering processes on Lick are reported by Brunetto et al. (2006a); different silicates were altered using a nanosecond pulsed UV laser in ablating conditions, producing spectral slopes higher than ion irradiation experiments. In these laser ablation experiments, the highest spectral slope was produced after laser ablation of San Carlos (Mg-rich) olivine. Also, a clear saturation effect was observed, as discussed by Brunetto et al. (2006a). Since Lick is an olivine rich asteroid, we plot in Fig. 1 

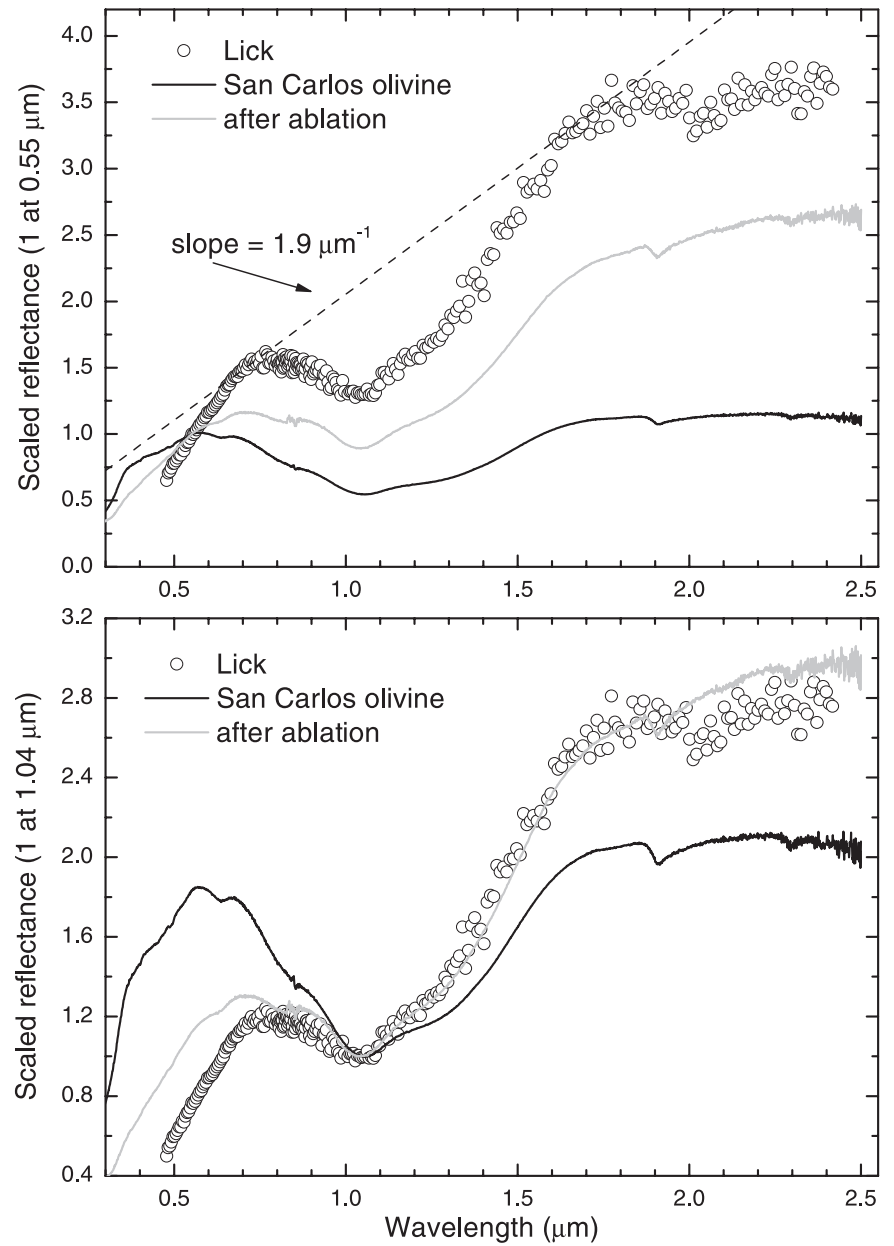

Fig. 1. The spectrum of Lick (circles) compared with the spectra of San Carlos olivine, before and after UV laser ablation. Upper panel: spectra are scaled at $0.55 \mu \mathrm{m}$; the dashed line represents the spectral slope. Lower panel: spectra are scaled at $1.04 \mu \mathrm{m}$.

a qualitative comparison of San Carlos olivine (before and after laser ablation) with Lick's spectrum.

The upper panel of Fig. 1, where spectra are scaled at $0.55 \mu \mathrm{m}$, indicates that Lick is distinctly redder than ablated olivine. The slope of Lick above the $1 \mu \mathrm{m}$ band is about $1.9 \mu \mathrm{m}^{-1}$. This high value of spectral slope puts Lick among the reddest Near-Earth asteroids, as can be deduced comparing with the slope distribution measured by Marchi et al. (2005); thus, it is a good test to check if the laboratory experiments can simulate space weathering at such a high level.

Since the highest slope variations are in the visible, it is more convenient to scale the spectra at $1.04 \mu \mathrm{m}$, i.e. close to the olivine band center. This is shown in the lower panel of Fig. 1. In this way one can force any model to reproduce the depth of the olivine band, and eventually understand what is causing the spectral mismatch in the continuum. It is clear that the spectrum of laser ablated olivine can reproduce the spectrum of Lick only for wavelength greater than about $0.8 \mu \mathrm{m}$. In fact, a large discrepancy is observed in the visible range.

\section{Results from spectral models}

To make the comparison between Lick's spectrum and laboratory data more quantitative, one can use the Hapke scattering model, that relies upon Hapke's description of radiative transfer

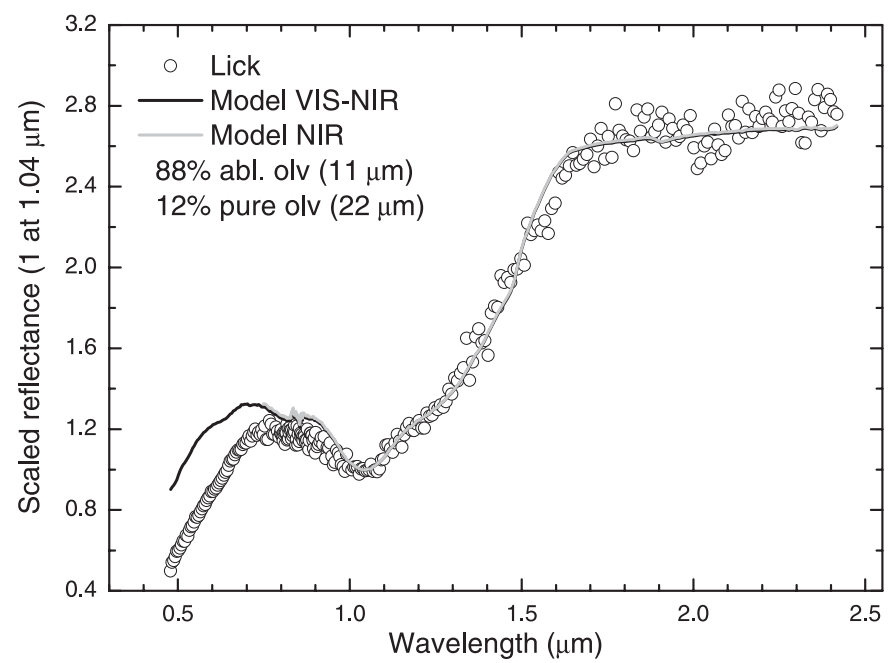

Fig. 2. Fit of Lick spectrum using optical constants of pristine and ablated olivine estimated by Brunetto et al. (2007). We consider intimate mixtures, using the Hapke scattering model. The gray line represents the best fit considering only the NIR range. Abundances and grain size are given for this mixture.

within particulate surfaces (Hapke 1981, 1993). In Fig. 2 it is shown a result obtained using such scattering model, combining only pure and ablated olivine; the corresponding optical constants were estimated by Brunetto et al. (2007). The result is unsatisfactory, because it can fit the $0.8-2.5 \mu \mathrm{m}$ range, but it cannot reproduce the visible range. It is interesting to note that the model restricted to the NIR gives a dominant percentage of weathered olivine with respect to the pristine one.

Lucey et al. (1998) studied the effects of temperature on the spectra of A-type asteroids. Temperature effects should not be the cause of the present discrepancies, because Near-Earth objects have surface temperature closer than Main-Belt asteroids to that of the experiments (room temperature). In fact, the comparison of the FWHM of Lick's olivine band with the plots of Lucey et al. (1998) indicates a reasonable surface temperature of about $200-300 \mathrm{~K}$.

Thus, discrepancies in the visible range could be caused by two different factors: (1) a space weathering effect; (2) a compositional effect.

Let us consider the space weathering effect first; it is plausible to investigate the possibility that the optical constants of ablated olivine are not adequate, because they do not represent the saturation regime. If this is the case, then one might be able to reproduce the visible spectrum of Lick using the Hapke space weathering model (small metallic iron inclusions in a silicate matrix) described by Hapke (2001), to check for a possible level of space weathering higher than the experimental one.

The Hapke's space weathering model is based on the Maxwell-Garnett effective medium theory, to calculate the absorption coefficient of a silicate host medium containing inclusions of small metallic iron spheres. The resulting absorption coefficient $\alpha(\lambda)$ of a material containing metallic iron particles is:

$\alpha(\lambda)=\alpha_{\mathrm{h}}(\lambda)+36 \pi \phi z(\lambda) / \lambda$

where $\lambda$ is the wavelength, $\alpha_{\mathrm{h}}(\lambda)$ is the absorption coefficient of the host silicate matrix, $\phi$ is the volume fraction of metallic iron particles in the host matrix, and it is a function of the 

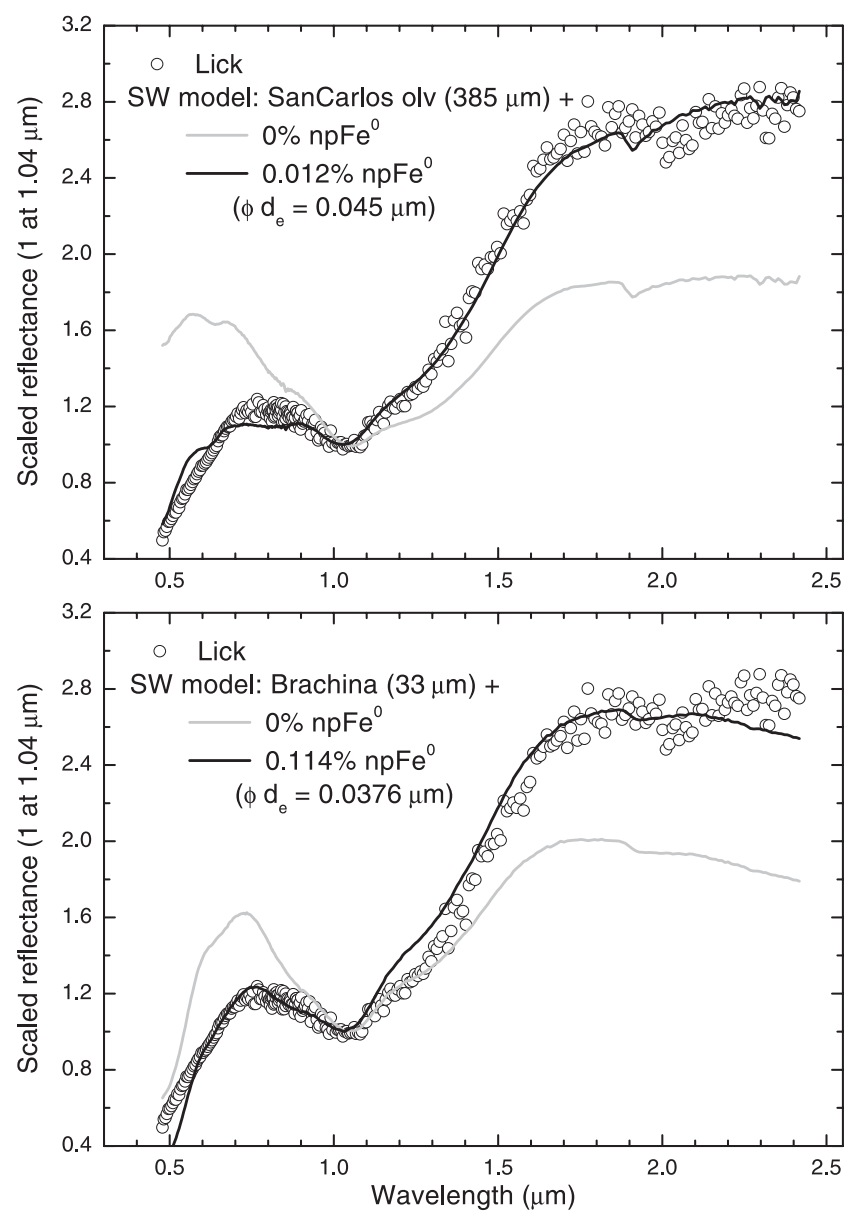

Fig. 3. Upper panel: fit of Lick spectrum using Hapke space weathering model (small metallic iron inclusions) and San Carlos olivine as starting composition. Lower panel: same as the upper panel, but using Brachina meteorite as starting composition. The grain size and the volume fraction of nanophase iron are also given in both panels.

concentration of inclusions and relative densities of inclusion and host materials. The function $z(\lambda)$ is given by:

$z(\lambda)=\frac{n_{\mathrm{h}}^{3} n_{\mathrm{Fe}} k_{\mathrm{Fe}}}{\left(n_{\mathrm{Fe}}^{2}-k_{\mathrm{Fe}}^{2}+2 n_{\mathrm{h}}^{2}\right)^{2}+\left(2 n_{\mathrm{Fe}} k_{\mathrm{Fe}}\right)^{2}}$

where $n_{\mathrm{h}}$ is the real part of the refractive index of the host material, $n_{\mathrm{Fe}}$ and $k_{\mathrm{Fe}}$ are the real and imaginary parts of the refractive index of iron. We use the optical constants of iron by Johnson \& Cristy (1974).

Applying Hapke's space weathering model to asteroid (25143) Itokawa, Hiroi et al. (2006) assumed that the absorbance spectrum of the asteroid can be approximated by the negative natural logarithm of its reflectance spectrum $R(\lambda)$ :

$\ln R(\lambda) \approx-\left\{\alpha_{\mathrm{h}}(\lambda)+36 \pi \phi z(\lambda) / \lambda\right\} d_{\mathrm{e}}$

where $d_{\mathrm{e}}$ is the mean optical path length. In the case $\phi=0$ (no iron inclusions), Eq. (3) gives an estimate of $\alpha_{\mathrm{h}}(\lambda)$ once we measure the reflectance spectrum and we have an estimate for the effective grain size. Although Eq. (3) is easy to use, it represents a relatively poor approximation. To apply Eq. (3) to an asteroid, we must start from the spectrum of a compound that has similar composition to the asteroid's one. Applying the metallic iron model, and using San Carlos olivine as starting composition, we obtain the results shown in the upper panel of Fig. 3 .
The fit (black curve) is better than Fig. 2, but some discrepancies are still present. Note, however, that the $\phi d_{\mathrm{e}}$ product is close to what what was found in the UV laser ablation experiments, i.e. about $0.045 \mu \mathrm{m}$ ( $\phi d_{\mathrm{e}}$ gives a measure of how red is the spectrum). In fact, Brunetto et al. (2007) found that the volume fraction of nanophase iron is $\sim 0.01 \%$ for ablated olivine (doses of 27 and $52 \mathrm{~J} / \mathrm{cm}^{2}$ ), with a mean optical path length of about $450 \mu \mathrm{m}$. Thus, the improvement of the upper panel of Fig. 3 probably comes from the peculiar combination of grain size and volume fraction of metallic iron inclusions.

Since discrepancies between observed and fit curves are only partially explained by a space weathering effect, it is worth to explore the compositional effect. Spectral mismatch could be explained by the presence of other components commonly found in silicate-rich asteroids, such as pyroxene. However, putting clinopyroxene or orthopyroxene in the scattering models does not help improving the fit.

The mineralogy of Lick can be compared with the one of the Brachinite and of the Pallasite meteorites (e.g. olivine-rich meteorites). In fact, after removing the continuum, the spectrum of Lick was found to be similar to the one of the Brachina meteorite (de León et al. 2004). This meteorite, that gives the name to the Brachinites group, is very rich in olivine (about $80 \%$ ) but it also includes plagioclase, clinopyroxene, chromite, Fe-sulfide, etc. (Mittlefehldt et al. 2003). Thus, if Lick's composition is similar to Brachina's, to get a good fit of the spectrum one should use all these components in the scattering models, not only olivine. Unfortunately, it is difficult to find optical constants for all those materials, and the ones available are often uncertain.

A solution comes from the use of Brachina's spectrum itself. From two RELAB (http://www.planetary.brown.edu/ $\mathrm{relab} /$ ) spectra at different grain size, we estimated the absorption coefficient, and then put it in the model (Eq. (3)), obtaining an effective grain size $d_{\mathrm{e}}$ for the asteroid (mean optical pathlength) and the volume fraction $\phi$ of nanophase iron. The result is shown in the lower panel of Fig. 3.

Although a mismatch is still present at around 0.5, 1.2, and $2.4 \mu \mathrm{m}$, the fit curve has an interesting trend, in particular in the visible range. The value $\phi d_{\mathrm{e}}=0.0376 \mu \mathrm{m}$ is close to the experimental saturation value, and this tells us that Lick is likely approaching a saturated space weathering effect. Discrepancies are present because probably the mineralogy of Brachina is not the same of Lick. From Fig. 3 it seems that San Carlos olivine can help in the spectral regions where the Brachina fit has discrepancies. Thus, performing a linear combination of the reflectance spectra shown in Fig. 3, we obtain the spectrum plotted in Fig. 4.

Note that in this case the observed spectrum is scaled using the IRAS albedo, while the fit curve is not scaled a priori, so that the model can output also an estimated geometric albedo. This makes the comparison more significant. Thus, a combination of $20 \%$ of San Carlos olivine and $80 \%$ of Brachina, with a nanophase iron model that produces $\phi d_{\mathrm{e}}=0.039 \mu \mathrm{m}$ (saturation regime), is able to well reproduce the spectrum and the albedo of Lick.

\section{Discussion}

The surface composition of Lick is probably comparable to Brachina's, but certainly with a higher content of Mg-rich olivine. In fact, San Carlos olivine has a $\mathrm{Mg}$ number of about 0.9, which is closer to the value that de León et al. (2004) found with the Modified Gaussian Model for Lick (Brachina has Mg number of about 0.66 ). 


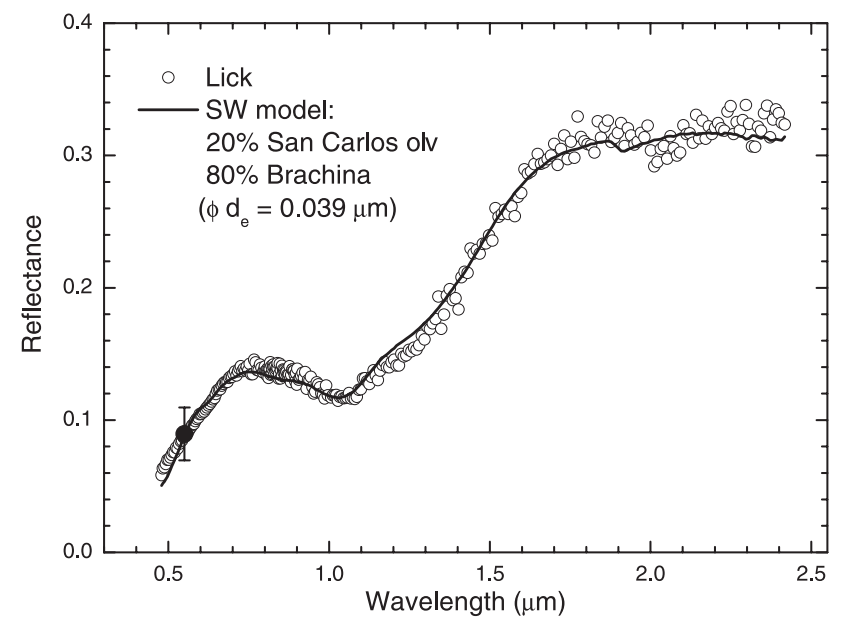

Fig. 4. Best fit of Lick spectrum obtained by a linear combination of the spectra plotted in Fig. 3. The final $\phi d_{\text {e }}$ product is about $0.039 \mu \mathrm{m}$. The IRAS albedo of this body is used to obtain a scale of absolute reflectance Tedesco et al. (2004).

Lucey et al. (1998) suggested that the A-type asteroids could be linked to the Pallasite meteorites, whose $\mathrm{Mg}$ number is in fact closer to what we estimate for Lick; unfortunately, we do not find a satisfactory fit using Pallasite spectra. However, our goal is to investigate the space weathering effect, while a detailed compositional analysis could be performed only if dedicated experiments on Brachinites and Pallasites were available. In other words, we do not claim that a direct link exists between Lick and the Brachinites. This point remains open for future studies.

Our nanophase iron model for Lick produces $\phi d_{\mathrm{e}}=$ $0.039 \mu \mathrm{m}$, similar to what Brunetto et al. (2007) found for laser ablated olivine. It is interesting to note that, on the contrary, the product $\phi d_{\mathrm{e}}$ obtained for ortho- and clinopyroxene by Brunetto et al. (2007) was about $0.02 \mu \mathrm{m}$. This is actually nearly identical to what Hiroi et al. (2006) found for the dark and red areas on asteroid Itokawa, whose composition is much more pyroxene-rich than Lick. This indicates that the laboratory experiments are a good simulation of the asteroidal space weathering.

An additional confirmation of the saturation regime comes from the comparison with the results of Brunetto et al. (2006b); it was found that computing the ratio between spectra of weathered and unweathered materials, the contribution of silicate bands almost disappears, and a continuum curve is left, that is related to the exposure to space weathering. This continuum is parameterized by a $C_{\mathrm{S}}$ coefficient, related to the exposure time. From Fig. 4, we estimate a value of $C_{\mathrm{S}} \sim-0.7 \mu \mathrm{m}$ for Lick. From the results of Brunetto et al. (2006b), we observe that this value is close to the saturation regime. As a comparison, asteroid (832) Karin (a recently rejuvenated body) has a value of $C_{\mathrm{S}} \sim-0.2 \mu \mathrm{m}$; the $C_{\mathrm{S}}$ parameter becomes more negative as the space weathering effect increases (Brunetto et al. 2006b).

It is hence clear that the surface of Lick is extremely weathered; this may give information about surface age and history, in particular about the lack of resurfacing events (micro-meteorites are believed to induce weathering, while macro-impactors are believed to rejuvenate the surface). Further investigation is required in terms of collisional evolution and impact events, but it exceeds the goal of this paper. However, based on the timescale estimated by Sasaki et al. (2001); Hapke (2001); Brunetto et al. (2006a), we can reasonably state that surface of Lick is probably older than about $10^{7}-10^{8}$ years, i.e. rejuvenating processes on this asteroid have been relatively rare in the last $10^{7}-10^{8}$ years. Interestingly, the radiation age of some Pallasite meteorites was found to be about $10^{8}$ years (Shukoliukov et al. 1991). However, we stress that future experiments should directly test in the laboratory the effects of weathering on the Brachinite and on the Pallasite meteorites to better constrain the timescales.

\section{Conclusions}

We compared the spectrum of A-type asteroid Lick with laser ablation experiments of silicate olivine. We applied the Hapke scattering and space weathering models to the asteroid spectrum, and successfully fit it in terms of slope, band profile, and albedo.

Results confirm that Lick has olivine with $\mathrm{Mg}$ number higher than that of the Brachina meteorite. The use of metallic iron inclusions in the model yields a product of volume fraction of inclusions times effective optical path length of about $0.039 \mu \mathrm{m}$, similarly to what observed experimentally after UV laser ablation of Mg-rich olivine, and differently from what has been measured for pyroxene in the laboratory, and in pyroxene-rich asteroids. The approach to a saturation regime (observed in the laboratory) seems the correct interpretation for an asteroid surface probably older than about $10^{7}-10^{8}$ years.

Acknowledgements. The authors wish to thank V. Orofino, T. Roush, and G. Strazzulla for their precious help and suggestions.

\section{References}

Bentley, M. S., Ball, A. J., Dyar, M. D., et al. 2005, 36th Annual Lunar and Planetary Science Conference, 36, 2255

Brunetto, R., Romano, F., Blanco, A., et al. 2006a, Icarus, 180, 546

Brunetto, R., Vernazza, P., Marchi, S., et al. 2006b, Icarus, 184, 327

Brunetto, R., Roush, T.L., Marra, A.C., \& Orofino, V. 2007, Icarus, in press

Bus, S. J., \& Binzel, R. P. 2002, Icarus, 158, 146

Chapman, C. R. 2004, Ann. Rev. Earth Planet. Sci., 32, 539

Cruikshank, D. P., \& Hartmann, W. K. 1984, Science, 223, 281

de León, J., Duffard, R., Licandro, J., \& Lazzaro, D. 2004, A\&A, 422, L59

Hapke, B. 1981, J. Geophys. Res., 86, 3039

Hapke, B. 1993, in Remote Geochemical Analysis: Elemental and Mineralogical Composition, ed. C. M. Pieters \& P. A. J. Englert (New York: Cambridge Univ. Press), 31

Hapke, B. 2001, J. Geophys. Res., 106, 10039

Hiroi, T., \& Sasaki, S. 2001, Meteorit. Planet. Sci., 36, 1587

Hiroi, T., Abe, M., Kitazato, K., et al. 2006, Nature, 443, 56

Johnson, P. B., \& Cristy, R. W. 1974, Phys. Rev. B, 9, 5056

Lucey, P. G., Keil, K., \& Whitely, R. 1998, J. Geophys. Res., 103, 5865

Marchi, S., Brunetto, R., Magrin, S., Lazzarin, M., \& Gandolfi, D. 2005, A\&A, 443, 769

Mittlefehldt, D. W., Bogard, D. D., Berkley, J. L., \& Garrison, D. H. 2003, Meteorit. Planet. Sci., 38, 1601

Pieters, C. M., Taylor, L. A., Noble, S. K., et al. 2000, Meteori. Planet. Sci., 35, 1101

Sasaki, S., Nakamura, K., Hamabe, Y., Kurahashi, E., \& Hiroi, T. 2001, Nature, 410,555

Shukoliukov, I. A., Klimenko, I. V., Koliasnikov, I. A., \& Petaev, M. I. 1991, Akademiia Nauk SSSR Doklady, 321, 825

Tedesco, E. F., Noah, P. V., Noah, M., \& Price, S. D. 2004, NASA Planetary Data System, IRAS-A-FPA-3-RDR-IMPS-V6.0, 12 